\title{
A CHARACTERIZATION OF GROWTH IN LOCALLY COMPACT GROUPS
}

\author{
BY J. W. JENKINS ${ }^{1}$
}

Communicated by Calvin C. Moore, July 31, 1972

$G$ will denote throughout a separable, connected, locally compact group. Fix a left Haar measure on $G$ and for a measurable subset $A$ of $G$, let $|A|_{G}$ denote the measure of $A$. The purpose of this note is to announce results concerning the asymptotic behavior of $\left|U^{n}\right|_{G}$ where $U$ is a compact neighborhood of the identity $e$ in $G$, and to indicate some of the applications these results have for various areas. The following definitions are required:

Definition 1. $G$ has polynomial growth if there is a polynomial $p$ such that for each compact neighborhood $U$ of $e$, there is a constant $C(U)$ so that

$$
\left|U^{n}\right|_{G} \leqq C(U) p(n) \quad(n=1,2, \ldots)
$$

$\left(U^{n}=\left\{u_{1} u_{2}, \ldots, u_{n} \mid u_{i} \in U, 1 \leqq i \leqq n\right\}\right) . G$ has exponential growth if for each compact neighborhood $U$ of $e$ there is a $t>1$ such that

$$
\left|U^{n}\right|_{G} \geqq t^{n} \quad(n=1,2, \ldots) .
$$

Note that since $G$ is connected, its "growth" will be determined by the behavior of $\left|U^{n}\right|_{G}$ for any one compact neighborhood $U$ of $e$.

For $a, b \in G$, let $[a, b]$ denote the subsemigroup of $G$ generated by $a$ and $b$, i.e.,

$$
[a, b]=\left\{x_{1} x_{2}, \ldots, x_{n} \mid x_{i} \in\{a, b\}, 1 \leqq i \leqq n, n=1,2, \ldots\right\} .
$$

$[a, b]$ is said to be free if $a[a, b] \cap b[a, b]=\varnothing$. A subset $S$ of $G$ is uniformly discrete if there is a neighborhood $U$ of $e$ in $G$ such that $s U \cap t U=\varnothing$ for $s, t \in S, s \neq t$.

Definition 2. $G$ is type $N F$ if there does not exist $a, b \in G$ such that $[a, b]$ is free and uniformly discrete.

Let $H$ be a connected Lie group with Lie algebra $\mathfrak{h}$, and let $g \rightarrow \operatorname{Ad} g$ be the canonical adjoint representation of $H$ on $\mathfrak{h}$. $H$ is said to be type $R$ if the eigenvalues of Adg are of absolute value one for each $g \in H$.

Since $G$ is connected, there exists an arbitrarily small compact normal subgroup $K$ of $G$ such that $G / K$ is a Lie group.

Definition 3. $G$ is type $R$ if there exists a compact normal subgroup $K$

AMS (MOS) subject classifications (1969). Primary 22.20, 22.50, 28.75.

${ }_{1}$ This research was partially supported by NSF Grants GP-28925 and GP-7952X3.

Copyright (C) American Mathematical Society 1973 
such that $G / K$ is a type $R$ Lie group.

THEOREM 4. The following conditions are equivalent:

(i) $G$ has polynomial growth,

(ii) $G$ is type $N F$,

(iii) $G$ is type $R$.

Outline of Proof. (i) $\Rightarrow$ (ii) is straightforward. To establish that (ii) $\Rightarrow$ (iii), we define groups $G_{\theta}$ for each $\theta=\theta_{1}+i \theta_{2}, \theta_{1}, \theta_{2} \in \boldsymbol{R}, \theta_{1} \neq 0$ and show that each $G_{\theta}$ is not type $N F$ and that, if $G$ is not type $R, G$ contains some $G_{\theta}$ as a topological subgroup. It then follows that $G$ is not type $N F$.

To show that (iii) $\Rightarrow$ (i), we first reduce to the case where $G$ is simply connected and solvable. One can then write $G=g_{1}\left(t_{1}\right) g_{2}\left(t_{2}\right) \cdots g_{n}\left(t_{n}\right)$ where each $g_{i}\left(t_{i}\right)$ is a one parameter subgroup of $G$. The argument proceeds by induction on $n$, using the fact that since $G$ is type $R,\left\|\operatorname{Ad} g_{i}(t)\right\|$ $\leqq p(t)$ for some polynomial $p$.

Comparison with discrete groups. Milnor [8] and Wolf [11] have investigated the growth of discrete solvable groups in connection with the study of fundamental groups of Riemannian manifolds with negative curvature. Combining their results with a recent result of Tits [10], one has the following: If $H$ is a linear group over a field $k$ with a finite set of generators $A=A^{-1}$, then (i) either $\left|A^{n}\right|_{H} \leqq p(n)$ for some polynomial $p$ and all $n \geqq 1$ or there is a $t>1$ such that $\left|A^{n}\right|_{H} \geqq t^{n}$ for all $n \geqq 1$, and (ii) if $\left|A^{n}\right|_{H}$ has polynomial growth, then $H$ is a finite extension of a solvable group $S$ and $S$ is a finite extension of a nilpotent group. We obtain analogous results for connected groups as a corollary to Theorem 4 .

COROLlaRY 5. (i) Either $G$ has polynomial growth or $G$ has exponential growth.

(ii) If $G$ is a connected Lie group with polynomial growth, then $G$ is the compact extension of a solvable Lie group $S$ and $\operatorname{Ad} S$ is an analytic subgroup of a compact extension of a nilpotent group.

REMARK. The first part of this corollary shows that in a connected group, a compact set cannot grow at a rate intermediate to polynomial and exponential, for example, such as $t^{n} / \log n$. This answers a question raised in Emerson and Greenleaf [4]. With regard to the second part, we remark that Hulanicki [5] has shown that a separable, locally compact group that is the compact extension of a nilpotent group cannot have exponential growth.

Strong amenability. In [4] Emerson and Greenleaf define a locally compact group $H$ to be strongly amenable if for every compact neighborhood $U=U^{-1}$ of $e$ in $H$ 


$$
\lim _{n}\left|U^{n+1}\right|_{H} /\left|U^{n}\right|_{H}=1
$$

Greenleaf has asked if every connected, amenable, unimodular group is necessarily strongly amenable. The following corollary to Theorem 4 provides a large class of counterexamples.

COROLlaRY 6. If $G$ is strongly amenable, then $G$ is type $R$. If $G$ is type $R$, then

$$
\lim _{n} \inf \left|U^{n+1}\right|_{G} /\left|U^{n}\right|_{G}=1
$$

for each compact neighborhood $U$ of $e$.

In particular, let $G$ be the semidirect product of $\boldsymbol{R}$ with $\boldsymbol{R}^{2}$ given by the homomorphism $\varphi: R \rightarrow \operatorname{Aut}\left(\boldsymbol{R}^{2}\right)$ where $\varphi(t)(x, y)=\left(e^{t} x, e^{-t} y\right)$ for $t \in \boldsymbol{R}$, $(x, y) \in \boldsymbol{R}^{2}$. Then $G$ is connected, amenable, unimodular but not type $R$, and hence, not strongly amenable.

An ergodic theorem. Let $X$ be a compact, separable metric space and assume $G$ is unimodular and has a jointly continuous action $G \times X \rightarrow X$ on $X$. A sequence of Borel subsets $\left\{A_{n}\right\}$ of $G$ is called balanced with respect to the action of $G$ on $X$ if $0<\left|A_{n}\right|_{G}<\infty$ for each $n$ and if whenever $\mu$ is a probability measure on $X$ invariant and ergodic under $G$ and $f \in C(X)$, the continuous complex valued functions on $X$, then

$$
\lim _{n}\left|A_{n}\right|_{G}^{-1} \int_{A_{n}} f\left(g \circ x_{0}\right) d g
$$

exist and equals $\int f d \mu$ for $\mu$-almost all $x_{0} \in X$.

An increasing sequence of subsets $\left\{A_{n}\right\}$ of $G$ grows evenly in $G$ if $0<\left|A_{n}\right|_{G}<\infty$ for each $n$,

$$
\lim _{k}\left|A_{k}\right|_{G}^{-1}\left|\left(A_{k} A_{n}\right) \Delta A_{k}\right|_{G}=0=\lim _{k}\left|A_{k}\right|_{G}^{-1}\left|\left(A_{n} A_{k}\right) \Delta A_{k}\right|_{G}
$$

for each $n$, and there is a constant $c>0$ such that $\left|A_{n}^{-1} A_{n}\right|_{G} \leqq c\left|A_{n}\right|_{G}$ for each $n$.

Calderón [3] and Bewley [2] have proved the following generalization of Birkhoff's individual ergodic theorem: If $G$ contains a sequence $\left\{A_{n}\right\}$ that grows evenly in $G$, then $\left\{A_{n}\right\}$ is balanced with respect to the action of $G$ on $X$.

Auslander and Brezin [1] have shown that any connected, simply connected, nilpotent Lie group $N$ contains a sequence of compact connected subsets that grow evenly in $N$. This is a special case of

COROLlary 7. If $G$ satisfies the equivalent conditions of Theorem 4 and 
if $U=U^{-1}$ is a compact neighborhood of the identity, then a subsequence of $\left\{U^{n} \mid n=1,2, \ldots\right\}$ grows unevenly in $G$.

On symmetry of $\mathscr{L}^{1}(G)$. A Banach *-algebra $\mathscr{U}$ is symmetric if $-x x^{*}$ is quasi-regular for each $x \in \mathscr{U}$, or equivalently by Raikov's Theorem [9], if

$$
(v(x)=) \lim _{n}\left\|x^{n}\right\|^{1 / n}=\sup \left\|T_{x}\right\| \quad(=\lambda(x))
$$

for each $x=x^{*} \in \mathscr{U}$, where the sup is taken over all *-representations $x \rightarrow T_{x}$ of $\mathscr{U}$. Hulanicki [5] has shown that if $H$ is a separable, locally compact group such that $\lim _{n}\left|A^{n}\right|_{H}^{1 / n} \leqq 1$ for any compact subset $A$ of $G$, then $v(x)=\lambda(x)$ for all $x=x^{*} \in \mathscr{L}^{1}(H)$ with compact support. Thus, any group with polynomial growth "almost" has a symmetric group algebra. (Observe that symmetry fails in this case only when the spectral radius is not continuous, and it is not known if this can ever occur in a group algebra.)

On the other hand, if $H$ is a discrete group, $l^{1}(H)$ is not symmetric if $H$ contains a free semigroup $[a, b]$ (cf. Jenkins [6]). There is evidence that suggests a similar statement obtains if $G$ is not type $N F$. Theorem 4 , therefore, lends support to a conjecture this author originally stated in [7], to wit, $\mathscr{L}^{1}(G)$ is symmetric if, and only if, $G$ is type $N F$.

Proofs of these and related results will appear elsewhere. This author wishes to express his thanks to R. Howe for many helpful suggestions related to this work.

\section{REFERENCES}

1. L. Auslander and J. Brezin, Uniform distributions in solvmanifolds, Advances in Math. 7 (1971), 111-144.

2. T. Bewley, Extension of the Birkhoff and von Neumann ergodic theorems to general semi-groups, Ann. Inst. Henri Poincaré 7 (1971), 283-291.

3. A. P. Calderón, A general ergodic theorem, Ann. of Math. (2) 58 (1953), 182-191. MR 14, 1071.

4. W. R. Emerson and F. P. Greenleaf, Asymptotic behavior of products $C^{p}=C+\cdots$ $+C$ in locally compact abelian groups, Trans. Amer. Math. Soc. 145 (1969), 171-204. MR 40 \# 2780.

5. A. Hulanicki, On positive functionals on a group algebra multiplicative on a subalgebra, Studia. Math. 37 (1971), 163-171.

6. J. Jenkins, On the spectral radius of elements in a group algebra, Illinois J. Math. 15 (1971), 551-554.

7. , Nonsymmetric group algebras, Studia Math. (to appear).

8. J. Milnor, Growth of finitely generated solvable groups, J. Differential Geometry 2 (1968), 447--449. MR 39 \#6212.

9. C. E. Rickart, General theory of Banach algebras, University Series in Higher Math., Van Nostrand, Princeton, N.J., 1960. MR 22 \#5903.

10. J. Tits, Free subgroups in linear groups (to appear).

11. J. A. Wolf, Growth of finitely generated solvable groups and curvature of Riemannian manifolds, J. Differential Geometry 2 (1968), 421-446. MR 40 \# 1939. 08540

School of Mathematics, Institute for Advanced Study, Princeton, New Jersey

Current address: Department of Mathematics, State University of New York, Albany, New York 12203 